\title{
Frecuencia de enfermedades de transmisión sexual en mujeres jóvenes
}

\author{
Clara Inés de Vargas*; Rosanna de Estrada**; Fernando de la Hoz***; Elizabeth Castañeda****
}

\begin{abstract}
RESUMEN: Debido a la frecuencia con que se presentan las enfermedades de transmisión sexual entre las personas jóvenes y las dificultades para su diagnóstico, estudiamos a 228 jóvenes universitarias, que presentaban síntomas vaginales, para conocer la frecuencia de Chlamydia trachomatis, Neisseria gonorrhoeae, Candida albicans, Trichomonas vaginalis y Gardnerella vaginalis. Adicionalmente, quisimos conocer si la actividad sexual o los métodos de planificación familiar constituían un factor de riesgo para estas infecciones. Al analizar los datos encontramos vaginosis bacteriana en el $31 \%$, vaginitis por Candida albicans en el $15 \%$ y cervicitis por Chlamydia trachomatis en el $22 \%$. Los métodos de planificación fueron un factor de riesgo para las diferentes infecciones, pero la actividad sexual no. No hubo una buena correlación entre los hallazgos clínicos y los de laboratorio, pero confirmamos la utilidad de los exámenes directos en la candidiasis y la vaginosis bacteriana, así como la necesidad de realizar exámenes de laboratorio específicos para el diagnóstico de la infección por $C$. trachomatis, infección muy frecuente, que causa gran morbilidad en las mujeres jóvenes en edad reproductiva.
\end{abstract}

PALABRAS CLAVES: Cervicitis, vaginitis, vaginosis, etiología.

SUMMARY: Due to the high frequency of sexually transmitted diseases among young people and the diagnostic difficulties that they present, we studied a group of $\mathbf{2 2 8}$ university students with vaginal symptoms in order to determine the frequency of isolation of Chlamydia trachomatis, Neisseria gonorrhoeae, Candida albicans, Trichomonas vaginalis and Gardnerella vaginalis. We also determined if sexual activity and contraception methods were risk factors for adquiring these infections. We found bacterial vaginosis in $31 \%$ of the studied population, $C$. albicans vaginitis in $15 \%$ and $C$. trachomatis cervicitis in $22 \%$. Contraception methods were the only risk factors found to be associated with infection. In the population studied we did not find a good correlation between clinical and laboratory findings; however, we confirmed the utility of direct examination in the diagnosis of bacterial vaginosis and candidiasis and the neccesity of specific diagnostic tests for the diagnosis of the chlamydial infections.

KEY WORDS: Cervicitis, vaginitis, vaginosis, etiology.

\section{Introducción}

Los cambios en el comportamiento humano, tales como el inicio temprano de las relaciones sexuales, el aumento del número de compañeros sexuales y el uso de anticonceptivos, son factores que han hecho que las enfermedades de transmisión sexual (ETS) y el número de agentes etiológicos que las ocasionan hayan aumentado y debido a la alta morbilidad que generan, estas entidades se han constituido en un importante problema de salud (1).

Por trabajos realizados en diferentes países, se considera que la infección por Chlamydia trachomatis, es una de las ETS más prevalentes, afectando entre el $8 \%$ y el $40 \%$ de las poblaciones estudiadas (2). Se estima que de 300 millones de casos en el mundo el $26 \%$ ocurre en menores de 20 años $(3,4)$; en la población universitaria de Estados Unidos la frecuencia informada es del 5 al $8 \%$ (2-7). Este microorganismo ha sido implicado como el causante del $40 \%$ de las uretritis no gonocóccicas, de cervicitis, enfermedad pélvica inflamatoria (EPI) y problemas en el embarazo e infertilidad (7-8).

\footnotetext{
* Bacterióloga grupo microbiología.Inst. Nal de Salud. Bogotá.

** Médico Grupo Microbiología. Inst. Nal de Salud. Bogotá.

*** Médico Grupo Epidemiología. Inst. Nal de Salud. Bogotá.

**** Microbióloga Grupo Microbiología. Inst. Nal de Salud. Bogotá.
}

Otra de las ETS de gran morbilidad, es la infección por Neisseria gonorrhoeae; la presencia de cepas productoras de beta lactamasa y el hecho de que cerca del $11 \%$ de las mujeres con gonorrea pueden ser asintomáticas y hacen que esta enfermedad sea de difícil control. La frecuencia de la enfermedad gonocóccica ha aumentado en los últimos años encontrándose en un $7 \%$ al $12 \%$ como agente etiológico de uretritis, cervicitis, EPI y oftalmia del recién nacido (9-11).

Existen otras ETS causantes de flujo vaginal, entre éstas la más frecuente es la vaginosis polimicrobiana causada entre otros gérmenes por Gardnerella vaginalis, esta vaginosis afecta del $25 \%$ al $30 \%$ de la población femenina, el hombre generalmente es asintomático pero sirve de reservorio $(7,12-13)$. Otra entidad muy frecuente es la candidiasis vulvo-vaginal, la cual es favorecida por el uso de anovulatorios, el embarazo y la inmunosupresión $(3,6,14)$.

La trichomoniasis es otra ETS, responsable del $20 \%$ de las vaginitis, en el hombre causa uretritis pero la infección puede ser asintomática, sirviendo en estos casos de reservorio $(3,14-15)$.

Uno de los problemas para el correcto diagnóstico y tratamiento de las ETS radica en que, en algunos casos, los signos clínicos y los exámenes directos realizados en el laboratorio tienen una baja sensibilidad y especificidad, 
por lo tanto se requiere del aislamiento del agente etiológico o de estudios inmunológicos que determinan antígenos o anticuerpos (3, 16-17).

De acuerdo con lo anterior, se diseñó un estudio transversal para conocer la frecuencia de las ETS por estos agentes, en una población de mujeres jóvenes, activas sexualmente y adicionalmente establecer el valor diagnóstico de los signos, síntomas clínicos y de los exámenes de laboratorio. También se consideró determinar si el número de compañeros sexuales, la frecuencia de las relaciones sexuales y los métodos de planificación familiar constituían factores de riesgo para adquirir esta patología.

En las 228 mujeres jóvenes sintomáticas encontramos, que los patógenos más frecuentes fueron la $C$. trachomatis en el cérvix y la $G$. vaginalis en la vagina; no se correlacionaron los hallazgos clínicos con los del laboratorio, pero confirmamos la utilidad del examen directo en el diagnóstico de la candidiasis y de la vaginosis bacteriana; el método anticonceptivo utilizado fue el único factor de riesgo para cada una de las infecciones.

\section{Material y métodos}

Pacientes. Se estudiaron 228 mujeres jóvenes activas sexualmente que consultaron, a un Servicio de Atención Médica Universitaria, entre marzo de 1988 a mayo de 1989 por presentar síntomas vaginales. Se excluyeron las pacientes que estaban en el primer y tercer trimestre del embarazo.

Se diseñó un formulario, el cual era diligenciado por la paciente en el cual debían consignar la edad, estado civil, número de embarazos, abortos espontáneos y provocados, número de partos, edad de la primera relación sexual, número de compañeros desde la primera relación, número de compañeros actuales y frecuencia de las relaciones sexuales por semana. También debían anotar los métodos anticonceptivos actuales, clasificados como dispositivos intrauterinos (DIU), anticonceptivos hormonales, barrera, condón o no uso y enfermedades de transmisión sexual previas de la paciente o de su compañero actual y los síntomas en el momento del ingreso al estudio.

En un formulario adicional, diligenciado por el médico, se anotó el resultado del examen ginecológico, se determinaron algunos signos clínicos como vulvitis, leucorrea en el introito, tipo de leucorrea, aspecto del cérvix: ectopia, quistes de Naboth, secreción purulenta y sangrado y $\mathrm{pH}$ vaginal determinado con papel $\mathrm{pH}$.

Toma de muestras. De cada paciente se tomaron con escobillón cuatro muestras del fondo de saco vaginal y dos muestras cervicales, éstas últimas fueron obtenidas una del orificio del canal cervical, empleando un escobillón con base de madera y la otra, empleando un escobillón con base de aluminio, el cual se rotó dentro del canal para obtener buena cantidad de células.

Procesamiento de las muestras. Las cuatro muestras del fondo de saco vaginal fueron procesadas de la siguiente manera: el primer escobillón se colocó en solución salina estéril para observar el microscopio la presencia de trofozoitos de $T$. vaginalis y realizar la prueba de las aminas con $\mathrm{KOH}$ al $20 \%$ (18); el material del segundo escobillón se sembró en una caja de agar Candida verde de bromocresol (Candida BGC, Difco) (19) para el aislamiento de Candida sp y con él también se hizo un extendido que se coloreó con Gram para observar las células guía, la presencia de blastoconidias y seudomicelios y la flóra bacteriana predominante $(12$, 18-19); el tercer escobillón se sembró sobre una caja con medio de agar sangre de cordero y otra con medio de agar sangre humana bifásico (HB) (18), para el diagnóstico de G. vaginalis y de gérmenes aerobios; el cuarto escobillón se sembró en un medio líquido selectivo para el cultivo de $T$. vaginalis (20).

La muestra tomada del orificio del canal cervical se sembró sobre una caja con medio de agar Thayer Martin, para el aislamiento de $N$. gonorrhoeae, se hizo un extendido y se coloreó con Gram para determinar la reacción leucocitaria y la presencia de diplococos intra y extracelulares $(9,21)$.

La segunda muestra cervical fue empleada para el cultivo de $C$. trachomatis, el escobillón fue colocado en un tubo con medio de transporte que consistía en RPMI adicionado con suero fetal bovino al $10 \%$ (Sigma) y antibióticos (gentamicina $10 \mathrm{mcg} / \mathrm{ml}$, vancomicina 100 $\mathrm{mcg} / \mathrm{ml}$ y anfotericina $\mathrm{B} 2 \mathrm{mcg} / \mathrm{ml}$ ). El medio con el escobillón se conservó a $-70^{\circ} \mathrm{C}$, hasta su procesamiento (22).

Identificación de gérmenes. La identificación de $C$. albicans a partir del cultivo se hizo con base en la prueba del tubo germinal y la fermentación de azúcares (19).

$\mathrm{La} G$. vaginalis fue identificada por su crecimiento en el medio HB, por la beta hemólisis después de una incubación a $37^{\circ} \mathrm{C}$ en $5 \%$ de $\mathrm{CO}_{2}$ durante 48 horas y por las pruebas bioquímicas. La identificación se correlacionó con la presencia de las células guía en el Gram y la prueba de aminas positiva en el fresco del flujo vaginal $(13,18)$.

El caldo para cultivo de $T$. vaginalis fue incubado a $37^{\circ} \mathrm{C}$ por siete días y una alícuota fue observada al microscopio diariamente para determinar la presencia de trofozoitos (20).

La identificación de $N$. gonorrhoeae se hizo a partir de las colonias crecidas sobre el Thayer Martin después de 48 horas de incubación a $37^{\circ} \mathrm{C}$ en $5 \%$ de $\mathrm{CO}_{2}$, con base en la coloración de Gram y las pruebas bioquímicas ( 9 , 21).

El cultivo para C. trachomatis se realizó sobre células McCoy, en microplacas de 96 cavidades de fondo plano (Costar), incubadas a $37^{\circ} \mathrm{C}$ en atmósfera de $5 \%$ de $\mathrm{CO}_{2}$ por 72 horas; la identificación del microorganismo se hizo demostrando la presencia de las inclusiones intracitoplasmáticas, ricas en glucógeno las cuales se colorean con yodo (22-23).

Análisis de datos. Se realizó un análisis univariado de los posibles factores de riesgo, comparando las frecuencias de cada factor entre las personas que fueron negativas y las que fueron positivas a todos los gérmenes investigados. La medida de asociación fueron los Odds Ratios (OR), en cuadros de cuatro casillas, calculándose los intervalos de 95\% (24). La sensibilidad (S), especificidad (E) y el valor predictivo positivo (VPP) de la correlación entre los hallazgos clínicos y los exámenes 
directos con los cultivos para C. trachomatis, G. vaginalis y C. albicans se determinó empleando la fórmula de Galen-Gambino (25).

También se hizo un análisis de tendencia para la asociación entre el método anticonceptivo y la infección por $C$. trachomatis, G. vaginalis y C. albicans, considerando como primer nivel de exposición el no haber usado ninguno, el segundo el uso de DIU, el tercero el condón, el cuarto los anticonceptivos hormonales, ya fueran orales o inyectados, y el quinto nivel, o de mayor riesgo, los métodos de barrera.

\section{Resultados}

Datos generales y del examen clínico. El formulario fue contestado por las 228 pacientes, pero en forma completa por $214(97 \%)$. Se determinó que 99\% tenían entre 15 a 30 años; $70 \%$ eran solteras, $18 \%$ casadas, $12 \%$ convivientes, una divorciada $(0,5 \%)$ y una viuda $(0,5 \%)$; $53 \%$ habían estado embarazadas, $35 \%$ habían tenido abortos provocados y $17 \%$ tenían de 1 a 3 hijos. Con relación a su experiencia sexual, $75 \%$ tuvieron su primera relación entre los 14 y los 20 años, 57\% había tenido más de un compañero desde el inicio de sus relaciones, pero $93 \%$ tenían en el momento del estudio sólo uno; la frecuencia de las relaciones sexuales por semana era de 1 a 2 en $71 \%$ y de tres o más en 28\%; 4 pacientes no contestaron a esta pregunta. En la Tabla 1 se observa la distribución de los datos generales en los diferentes grupos de edad.

Los métodos de planificación más usados fueron los dispositivos intrauterinos en $29 \%$ y los preservativos en $20 \%, 14 \%$ usaban tabletas vaginales y $10 \%$ anovulatorios orales o inyectados; $31,5 \%$ no planificaban y 14 de las pacientes no respondieron a esta pregunta.

Tabla 1

DISTRIBUCION DE ALGUNAS VARIABLES EN LAS PACIENTES ESTUDIADAS n=228

\begin{tabular}{|c|c|c|c|c|c|c|c|c|c|c|c|c|c|c|c|c|}
\hline \multirow[t]{3}{*}{ Edad } & \multirow{3}{*}{\multicolumn{2}{|c|}{$\begin{array}{l}\text { Total } \\
\mathrm{n} \quad(\%)\end{array}$}} & \multicolumn{6}{|c|}{ Estado Civil } & \multicolumn{2}{|c|}{ Embarazos } & \multicolumn{2}{|c|}{ Abortos } & \multicolumn{2}{|c|}{ Partos } & \multicolumn{2}{|c|}{$\mathrm{NCS}>1$} \\
\hline & & & \multicolumn{2}{|c|}{ Casada } & \multicolumn{2}{|c|}{ Soltera } & \multicolumn{2}{|c|}{ Conviviente } & \multirow{2}{*}{\multicolumn{2}{|c|}{$\mathrm{n} \quad(\%)$}} & \multirow{2}{*}{\multicolumn{2}{|c|}{$\mathrm{n} \quad(\%)$}} & \multirow{2}{*}{\multicolumn{2}{|c|}{$\mathrm{n} \quad(\%)$}} & \multirow[t]{2}{*}{$\mathrm{n}$} & \multirow[t]{2}{*}{$(\%)$} \\
\hline & & & & $(\%)$ & & $(\%)$ & & $(\%)$ & & & & & & & & \\
\hline $15-19$ & 16 & (7) & 1 & (3) & 14 & (9) & 1 & (4) & & $(2,5)$ & 2 & (3) & 1 & (3) & 9 & (7) \\
\hline $20-24$ & 139 & (61) & 18 & (47) & 108 & (68) & 13 & (46) & & $(62)$ & 55 & (69) & 17 & (44) & 74 & (57) \\
\hline $25-29$ & 67 & (29) & 18 & (47) & 34 & (21) & 14 & $(50)$ & & (34) & 23 & (28) & 19 & (49) & 45 & (35) \\
\hline $30-35$ & 6 & (3) & 1 & (3) & 4 & (2) & & & & $(2,5)$ & - & & 2 & (4) & 1 & (1) \\
\hline Total & 228 & (100) & 38 & (17) & 160 & (70) & 28 & (12) & 120 & (53) & 80 & (35) & 39 & (18) & 129 & (57) \\
\hline
\end{tabular}

NCS = Número de Compañeros Sexuales

Tabla 2

RESULTADOS DE LOS EXAMENES REALIZADOS EN LAS MUESTRAS VAGINALES DE 228 PACIENTES

\begin{tabular}{|lrr|}
\hline EXAMEN & $\mathrm{n}$ & $\%$ \\
\hline FRESCO: & 220 & 96 \\
pH > 4,5 & 51 & 22 \\
Aminas positivas & 2 & 1 \\
T. vaginalis & & \\
COLORACION DEL GRAM & 53 & 23 \\
Células guía & 35 & 15 \\
Blastoconidias & 31 & 14 \\
Seudomicelios & & \\
CULTIVOS & 71 & 31 \\
G. vaginalis & 34 & 15 \\
C. albicans & 2 & 1 \\
T. vaginalis & 91 & 40 \\
Negativos & \\
\hline
\end{tabular}

Antecedentes de ETS. La trichomoniasis fue la ETS más frecuente en las pacientes (10\%) y la gonorrea en sus compañeros $(2 \%)$; el $10 \%$ de las pacientes había sufrido un episodio de EPI.

Sintomatología. El síntoma clínico más frecuente fue el flujo vaginal en $95 \%$ de las pacientes, acompañado de escozor en $42 \%$, dolor al coito en $32 \%$, aumento del número de micciones en $27 \%$ y ardor en $21 \%$. Se observó que $28 \%$ de las pacientes presentaban únicamente flujo vaginal.

Examen clínico. Al examen clínico de la vagina $93 \%$ tenían flujo vaginal, $43 \%$ con aspecto grumoso; $60 \%$ presentaron leucorrea en el introito y $38 \%$ vulvitis. En el cérvix $18 \%$ presentaron secreción purulenta, el cérvix era friable en $43 \%$ y estaba erosionado en $62 \%$; el $17 \%$ presentaron el cérvix sano.

Hallazgos del laboratorio. En la Tabla 2 se anotaron los resultados de los exámenes realizados en el laboratorio a partir de las muestras vaginales. En la Tabla 3 se resumen los resultados de los exámenes directos y cultivos cervicales. Los cultivos de 91 (40\%) pacientes fueron negativos tanto en vagina como en cérvix. 
Correlación de los hallazgos clínicos y de laboratorio. En la Tabla 4 se correlacionan los hallazgos clínicos y de laboratorio con el diagnóstico de la vaginosis bacteriana, establecida por el cultivo de G. vaginalis, y se presentan para estos parámetros la sensibilidad, la especificidad y el VPP. Al hacer una asociación del aislamiento de G. vaginalis, con el flujo gris, la presencia de células guía y la prueba de aminas positiva se encontró el cultivo positivo en el $94,3 \%$ de las pacientes que tenían 2 de estos parámetros y en el $100 \%$ de las que tenían los 3 ; el cultivo fue negativo en $5,7 \%$ de las pacientes con 2 de estos parámetros.

En la Tabla 5 se relacionan los hallazgos clínicos y de laboratorio con el diagnóstico de la candidiasis vaginal establecida por el cultivo de $C$. albicans y se presenta la sensibilidad, la especificidad y el VPP de los hallazgos. Se determinó como vaginitis la presencia de uno o varios síntomas como vulvitis, escozor, ardor y/o dolor al coito y se encontró en 30/34 (88\%) pacientes con cultivos positivos para $C$. albicans y en 51/91 (56\%) pacientes con cultivos negativos.

La Tabla 6 muestra la relación de los hallazgos clínicos y de laboratorio en el diagnóstico de la cervicitis por $C$. trachomatis diagnosticada por cultivo y la sensibilidad, la especificidad y el valor predictivo positivo para cada uno de estos hallazgos. Es de anotar que 11 $(22 \%)$ de las pacientes con cultivo positivo para $C$. trachomatis tenían el cérvix sano.

Se consideró como cervicitis la presencia de una secreción purulenta, friabilidad, ectopia y/o erosión y se estableció en $38 / 49$ (78\%) pacientes con cultivo de $C$. trachomatis y en 66/91 (73\%) pacientes con cultivo negativo; no se encontró diferencia significativa entre los dos grupos de pacientes.

Factores de riesgo.Entre las variables analizadas se encontró que los métodos de planificación familiar fueron los únicos que representaron un factor de riesgo.

Al realizar el análisis de tendencia para los Odds Ratio a los diferentes métodos de planificación familiar, en la infección por $G$. vaginalis se determinó un Riesgo Relativo (RR) de 7,5 al comparar el método de barrera con el uso de DIU y de 5,8 entre las que usaban método de barrera comparado con las que no usaban ningún método $(\mathrm{P}<0,007)$.

En las pacientes con infección por $C$. albicans encontramos en el análisis de tendencia, que las pacientes que utilizaban métodos de barrera y las que utilizaban anticonceptivos hormonales tenían 12 y 4 veces mayor riesgo de infección $(\mathrm{P}<0,003)$ comparadas con las que no utilizaban ningún método o las que utilizaban DIU.

En las pacientes con infección por $C$. trachomatis se observó un gradiente de tendencia ascendente para los riesgos relativos a través de los niveles de exposición, encontrándose que las mujeres que usaban los métodos de barrera tenían 16 veces más riesgo de estar infectadas por este microorganismo y de 3 veces el riesgo cuando utilizaban anticonceptivos hormonales $(\mathrm{P}<0,001)$, comparado con el no uso de anticonceptivos o el uso de DIU.

Tabla 3

RESULTADOS DE LOS EXAMENES

REALIZADOS EN LAS MUESTRAS CERVICALES DE 228 PACIENTES

\begin{tabular}{|lrr|}
\hline EXAMEN & $\mathrm{n}$ & $\%$ \\
\hline COLORACION DE GRAM & & \\
PMN > $10 \times$ cm 1000X & 58 & 25,0 \\
& & \\
CULTIVOS & & \\
C. trachomatis & 49 & 22,0 \\
N. gonorrhoeae & 1 & 0,4 \\
Negativos & 91 & 40,0 \\
\hline
\end{tabular}

Tabla 4

\section{RELACION DE LOS HALLAZGOS CLINICOS Y DE LABORATORIO EN EL DIAGNOSTICO DE LA VAGINOSIS BACTERIANA EN 71 PACIENTES CON CULTIVO POSITIVO PARA GARDNERELLA VAGINALIS}

\begin{tabular}{|lccccc|}
\hline Clínica-Examen directo & $\begin{array}{c}\text { Total } \\
\text { Pacientes } \\
\text { Hallazgo }\end{array}$ & $\begin{array}{c}\text { Total } \\
\text { Pacientes } \\
\text { Cult +/Hallazgo }\end{array}$ & S & E & VPP \\
\hline SIGNOS: & & & & & \\
Flujo en el introito & 137 & 44 & 62 & 41 & 32 \\
Flujo gris & 55 & 21 & 30 & 78 & 32 \\
Flujo grumoso & 104 & 33 & 46 & 45 & 31 \\
EXAMEN EN FRESCO & & & & 4 & 80 \\
pH > 4,5 & 220 & 69 & 97 & 94 & 91 \\
Prueba Amina (positiva) & 51 & 41 & 58 & 98 & \\
Células Guía (positiva) & 53 & 48 & 68 & & \\
\hline
\end{tabular}


Tabla 5

RELACION DE LOS HALLAZGOS CLINICOS Y DE LABORATORIO EN EL DIAGNOSTICO DE CANDIDIASIS VAGINAL EN 34 PACIENTES CON CULTIVO POSITIVO PARA CANDIDA ALBICANS

\begin{tabular}{|lccccc|}
\hline Clínica-Examen directo & $\begin{array}{c}\text { Total } \\
\text { Pacientes } \\
\text { Hallazgo }\end{array}$ & $\begin{array}{c}\text { Total } \\
\text { Pacientes } \\
\text { Cult. +/Hallazgo }\end{array}$ & S & E & VPP \\
\hline SIGNOS: & 104 & 22 & 65 & 58 & 21 \\
Flujo grumoso & 86 & 22 & 65 & 67 & 26 \\
Vulvitis & & & & 62 & 23 \\
SINTOMAS: & 95 & 22 & 65 & & 69 \\
Escozor & & & & 94 & 71 \\
COLORACION DE GRAM: & 35 & 24 & 71 & 95 & \\
Blastoconidias & 34 & 22 & 65 & & \\
Seudomicelios & & & & & \\
\hline
\end{tabular}

Tabla 6

RELACION DE LOS HALLAZGOS CLINICOS Y DE LABORATORIO EN EL DIAGNOSTICO DE CERVICITIS POR CHLAMYDIA TRACHOMATIS EN 49 PACIENTES CON CULTIVO POSITIVO

\begin{tabular}{|lccccc|}
\hline Clínica-Examen directo & $\begin{array}{c}\text { Total } \\
\text { Pacientes } \\
\text { Hallazgo }\end{array}$ & $\begin{array}{c}\text { Total } \\
\text { Pacientes } \\
\text { Cult. +/Hallazgo }\end{array}$ & S & E & VPP \\
\hline SIGNOS: & & & & & \\
Secreción purulenta cervical & 41 & 5 & 43 & 57 & 12 \\
Cérvix friable & 98 & 21 & 24 & 85 & 32 \\
Ectopia & 38 & 12 & 71 & 41 & 25 \\
Erosión & 141 & 15 & 25 & 25 \\
Quistes de Naboth & 4 & 15 & 31 & 87 & 26 \\
GRAM CERVICAL & 38 & & & & \\
$>$ 10 PMN x cm 100X & & & & & \\
\hline
\end{tabular}

\section{Discusión}

El grupo de jóvenes sexualmente activas estudiadas fue heterogéneo, el alto porcentaje de abortos provocados nos confirma lo planteado en la Encuesta Nacional de Salud, al señalar, el aborto provocado, como la principal causa de pérdida del producto en el primer trimestre del embarazo (26). El promedio de hijos por edades o por estado civil se encontró por debajo a lo informado como promedio en el país, lo cual se explica por ser una población universitaria (26).

En cuanto a la actividad sexual encontramos que la edad del inicio de las relaciones sexuales es semejante a la informada en otros estudios (4), no obstante determinarse más de un compañero sexual en el $60 \%$ de ellas, no se pudo establecer el tiempo que llevaban con el último compañero, para considerarse este tiempo como factor de riesgo.
Referente al método de planificación, en el 70\% q'ue la utilizaba, llama la atención el pequeño porcentaje que planificaba con anticonceptivos hormonales o con DIU y el poco uso del condón, a pesar de las campañas para el uso de este último en la prevención del SIDA y de otras ETS.

Se informaron muy pocos antecedentes de ETS tanto en los compañeros como en ellas mismas, pero esto pudo deberse al desconocimiento de estas enfermedades, sobre todo aquellas con un alto porcentaje de formas asintomáticas.

En los hallazgos de laboratorio es importante destacar que todas las pacientes eran sintomáticas y el flujo de casi todas tenía un $\mathrm{pH}>5.0$, similar a los datos obtenidos en otros estudios, por lo tanto este parámetro fue considerado el más sensible pero el menos específico, dentro de los cuatro criterios establecidos para el diagnóstico de vaginosis bacteriana (27). 
Similar a lo informado en otros estudios, la G. vaginalis se constituyó en el germen aislado con mayor frecuencia en la vagina, seguido por $C$. albicans $(6,15)$. En el cérvix C. trachomatis fue el microorganismo implicado como causante de cervicitis, confirmando lo descrito en otras poblaciones estudiadas en nuestro medio (6-7) y en numerosos informes en la literatura (1-5). El aislamiento de $T$. vaginalis de la vagina y de $N$. gonorrhoeae de cérvix fue escaso, con resultados similares a otros grupos estudiados en nuestro laboratorio $(6,15)$.

En las pacientes con vaginosis bacteriana la sensibilidad de las células guía en el flujo vaginal no fue muy alta, pero tuvo muy buena especificidad, lo que nos indica que la ausencia de estas células no descarta una vaginosis bacteriana, pero su presencia es un importante criterio paraclínico para su diagnóstico (27); además, según estudios de varios autores, las células guía deben observarse en fresco, para evitar el daño o la pérdida de la célula al realizar el extendido para colorear con Gram (27-28).

La prueba de aminas y la apariencia del flujo vaginal, presentaron una baja sensibilidad debido posiblemente, a que estos signos están sujetos a una interpretación subjetiva y a factores fisiológicos (27).

Eschembach y Nuget consideran que debido a que los signos clínicos de la vaginosis bacteriana son difíciles de unificar y no son muy específicos, es necesario realizar exámenes de laboratorio para confirmar su diagnóstico (27-28); ellos recomiendan utilizar el examen directo coloreado con Gram, para observar el cambio de la flora bacteriana vaginal y el examen en fresco para determinar la presencia de las células guía y determinar la presencia de las aminas, logrando una mejor sensibilidad y especificidad en el diagnóstico (27-28).

En el diagnóstico de la vaginitis por $C$. albicans, los signos y síntomas clínicos tuvieron una baja especificidad, mostrando la necesidad de realizar exámenes de laboratorio para hacer un diagnóstico.

Los parámetros de sensibilidad, especificidad y VPP de la secreción purulenta, los signos de cervicitis y la reacción leucocitaria > $10 \mathrm{PMN}$ x cm (1000X) para la infección por C. trachomatis fueron muy bajos y similares a los informados por Nugent, éste y otros autores recomiendan realizar cultivos o determinar la presencia del antígeno para establecer el diagnóstico de esta infección (16, 29-30); además consideran que los signos y síntomas de cervicitis, pueden deberse a una reacción del epitelio columnar cuando es expuesto a factores no infecciosos, a displasia cervical o a una salpingitis aguda y que el $50 \%$ de las pacientes con secreción mucopurulenta no tienen infección por $C$. trachomatis o por $N$. gonorrhoeae (29-30).

Contrario a lo encontrado en otros estudios, con poblaciones similares, la edad, los años de relaciones sexuales y la friabilidad o ectopia del cérvix, no fueron factores de riesgo para la infección por $C$. trachomatis (4). La asociación puede ser subestimada, porque el grupo control (pacientes con cultivo negativo) pudo no ser adecuado dado que todas las participantes tenían síntomas vaginales. Adicionalmente otras patologías de transmisión sexual no detectadas por el cultivo, pudieron estar presentes en el grupo control.

Siempre se han descrito los anovulatorios como el método anticonceptivo que presenta mayor riesgo para contraer la infección por $C$. trachomatis, debido al incremento del tejido columnar y del tejido ectópico, por los cuales la C. trachomatis tiene predilección (5); sin embargo en nuestro estudio los anovulatorios no se comportaron como factor de riesgo. Vale la pena anotar que la frecuencia del uso de anovulatorios fue muy baja lo cual pudo influir en la no asociación con la infección por $C$. trachomatis. Al hacer el análisis de tendencia el mayor riesgo estuvo en los métodos de barrera los cuales han sido considerados como protectores (4).

Con lo mencionado podemos concluir que en la población joven estudiada la frecuencia de las ETS determinadas es similar a la de otras poblaciones (6-7) y que es necesario emplear las pruebas de laboratorio para determinar la verdadera etiología del flujo vaginal y así permitir que la paciente y su compañero reciban el tratamiento adecuado para evitar la diseminación de estas enfermedades.

\section{BIBLIOGRAFIA}

1. Holmes KK., Bell TA., Berger RE. Epidemiology of sexually trasmitted diseases. Urol. Clin. North. Am. 1984; 11: 3.

2. Centers for disease control. Chlamydia trachomatis infections: policy guidelines for prevention and control. MMWR: 1985; 34 (suppl) 53s.

3. Mc Cormack WM., Rosner B., Mc Comb DE., Zinner SH. Infection with Chlamydia trachomatis in female college students. Am. J. Epidemiol. 1985; 121: 107.

4. Harrison R., Costin M., Meder J., Bownds L., Sin D., Lewis M., Alexander R. CervicalChlamydia trachomatis infections in University women: Relationship to history, contraception, ectopy and cervicitis. Am. J. Obstet. Gynecol. 1985; 153: 244.

5. Shafer MA., Beck A., Blain B., Dole P., Irwin CE., Sweet R., Schachter J. Chlamydia trachomatis: Important relationships to race, contraception, lower genital tract infection, and Papanicolau smear. J. Pediatrics 1984; 104: 141.

6. Heredia R., Agudelo CI., Castañeda E. Prevalencia de los agentes etiológicos de la vaginitis y cervicitis en pacientes de la consulta ginecológica general. Acta. Med. Colomb. 1990; 15: 92.
7. Vargas CI., Heredia R., Castañeda E. Infección por Chlamydia trachomatis en pacientes con neoplasia intracervical. Rev. Colomb. Obstet. y Ginecol. 1990; 42: 99.

8. Schachter J. Chlamydial infections. N. Engl. J. Med. 1978; 298: 428.

9. Morello J., Janda W., Bohnoff M. Neisseria and Branchamella In: Manual of Clinical Microbiology. Fifth Edition. A balows, WJ Hausler, KL Herrman, HD Isemberg, HJ Shadomy (Eds). American society for Microbiology. Washington DC. 1991; 258.

10. Dallbetta G., Hook ED. Gonococcal Infections. Infect. Dis. Clin. of North. Amercia 1987; 1: 25.

11. Center for Disease Control. Antibiotic resistant strains of Neisseria gonorrhoeae: Policy guidelines for detection, management and control. MMWR: 1987; 36 (Suppl). 1S.

12. Spiegel C. Bacterial vaginosis. Clin. Microbiol. Rev. 1991; 4: 485.

13. Catlin BW. Gardnerella vaginalis: Characteristics, clinical considerations, and controversies. Clin. Microbiol. Rev. 1992; 5: 213. 
14. Díaz F., Vélez H., Vélez MI., Jiménez OL., Ochoa SI. Flujo vaginal. Experiencia con el diagnóstico microbiológico de 1178 casos. Biomédica 1985; 5: 92.

15. Vargas CI., Galindo BL., Martínez JE. Vaginitis y cervicitis en planificación familiar. Rev. Colomb. de Obstet. y Ginecol. 1991; 42: 223.

16. Moscicki B., Shafer MA., Millstein SG., Irwin CE., Schachter J. The use and limitations of cervical Gram stains and mucopurulent cervicitis as predictors for Chlamydia trachomatis in female adolescents. Am. J. Obstet. and Gynecol. 1987; 157: 65.

17. Ehret JM., Judson FN., Biddle J. Gonorrhea In: Laboratory Methods for the diagnosis of sexually transmitted diseases. BB Wentworth, FN Judson (Eds). American Pubic Health Association. Washington DC $1984 ; 43$.

18. Vargas CI., Vanegas D., Heredia R. Gardnerella vaginalis. Aspectos clínicos y de laboratorio. Serie de Notas e Informes Técnicos No. 18. Bogotá, Instituto Nacional de Salud 1989.

19. Campell MC., Stewart JL., Larsh HW. The Medical Mycology Handbook. E. Tommaso, M. Zirke (Ed.) 1980.

20. Schmid GP., Matheny LC., Zaidi AA., Kraus SJ. Evaluation of six media for the growth of Trichomonas vaginalis from vaginal secretions. J. Clin. Microbiol. 1989; 27: 1230.

21. Guzmán M., Vargas CI.Neisseria. En Microbiología Médica. Manual de procedimientos. Serie de Publicaciones Científicas No. 14 Bogotá, Instituto Nacional de Salud 1988; 127.
22. Schachter J. Chlamydiae In Manual of Clinical Microbiology Fifth edition. A. Balows, KL Herrmann, HD Isemberg, HJ Shadomy (Eds). ASM Washington DC 1991; 1045.

23. Bird BR., Forrester ET. Laboratory diagnostic of Chlamydia trachomatis infections. U.S. Department of Health and Human Services Center for Disease Control. Atlanta. EA 1982.

24. Rothman KJ. Medidas del efecto En: Epidemiología Moderna. Ediciones Díaz de Santos SA. Madrid 1987; 43.

25. Galen RS., Gambino SR. Beyond normality: The predictive value and efficiency of medical diagnoses. New York. John Wiley and Sons; $1975 ; 10$.

26. Carmona F. Mujer y reproducción humana. Colombia 1886-1989. Encuesta Nacional de Conocimientos, Actitudes y Prácticas en Salud. Instituto Nacional de Salud. Bogotá, 1990.

27. Eschembach DA., Hiller S., Critchlow C., Stevens C., DeRouen T. Holmes KK. Diagnosis and clinical manifestations of bacterial vaginosis. Am. J. Obstet. Gynecol. 1988; 158: 819-828.

28. Nuget RP., Krohn MA., Hiller SL. Reliability of diagnosing bactrial vaginosis is improved by a standardized method of Gram stain interpretation. J. Clin. Microb. 1991; 29(2): 297-301.

29. Nuget RP., Hiller SL. Mucupurulent cervicitis as predictor of chlamydial infection and adverse pregnancy outcome. Sex Trans Diseas. 1992; 19: 198-202.

30. Paavonen J., Critchlow CW., Derouen T et al. Etiology of cervical inflammation. Am. J. Obstet. Gynecol. 1986; 154: 556-564.

\section{Correos}

\section{de Colombia}

\section{Adpostal}

\section{Estos son nuestros servicios ¡utilícelos!}

- SERVICIO DE CORREO ORDINARIO

- SERVICIO DE CERTIFICADO ESPECIAL

- ENCOMIENDAS CONTRA REEMBOLSO

- SERVICIO DE FILATELIA

- SERVICIO ELECTRONICO

- SERVICIO INTERNACIONAL APR/SAL

- SERVICIO RESPUESTA COMERCIAL

- SERVICIOS ESPECIALES
- SERVICIO DE CORREO CERTIFICADO

- SERVICIO ENCOMIENDAS ASEGURADAS

- SERVICIO CARTAS ASEGURADAS

- SERVICIO DE GIROS

- BUROFAX

- SERVICIO "CORRA"

- SERVICIO TARIFA POSTAL REDUCIDA

Teléfonos para quejas y reclamos: 3340304 - 3415536 - Santafé de Bogotá, D.C. - Colombia

\section{Cuente con nosotros hay que creer en los Correos de Colombia}

